\title{
Total hip arthroplasty for Crowe IV hip without subtrochanteric shortening osteotomy -a long term follow up study
}

\author{
Toshiyuki Kawai ${ }^{1,2^{*}}$, Chiaki Tanaka ${ }^{1}$ and Hiroshi Kanoe ${ }^{1}$
}

\begin{abstract}
Background: Several authors reported encouraging results of total hip arthroplasty (THA) for Crowe IV hips performed using shortening osteotomy. However, few papers have documanted the results of THA for Crowe IV hips without shortening osteotomy. The aim of the present study was to assess the long term-results of cemented THAs for Crowe group IV hips performed without subtrochanteric shortening osteotomy.

Methods: We have assessed the long term results of 27 cemented total hip arthroplasty (THA) performed without subtrochanteric osteotomy for Crowe group IV hip. All THAs were performed via transtrochanteric approach.

Results: After a mean follow-up of 10.6 (6 to 17.9) years, 25 hips (92.6\%) had survived without revision surgery and survivorship analysis gave a survival rate of $96.3 \%$ at 10 years with any revision surgery as the end point. Although mean limb lengthening was $3.2(1.0$ to 5.1$) \mathrm{cm}$, no hips developed nerve palsy. Complications occurred in four hips, necessitating revision surgery in two. Among the four complications, three involved the greater trochanter, two of which occurred in cases where braided cables had been used to reattach the greater trochanter.

Conclusions: Although we encountered four complications, including three trochanteric problems, our findings suggest that THA without subtrochanteric shortening osteotomy can provide satisfactory long-term results in patients with Crowe IV hip.
\end{abstract}

\section{Background}

Total hip arthroplasty (THA) is an effective operation to relieve pain and improve function in an osteoarthritic hip [1]. Charnley suggested that untreated congenital dislocation of the hip is a contraindication for THA because of a lack of acetabular bone stock [2], raising concern about the higher rate of aseptic loosening of the acetabular component in subluxed hips.

Placement of the acetabular component in the true acetabulum has yielded the most durable results of THA in patients with developmental dysplasia of the hip [3-6]. In Crowe group IV hips, however, anatomic socket placement can make hip reduction difficult, and reduction may cause considerable limb lengthening and increase the risk of neurologic traction injury [2,7]. Therefore, femoral

\footnotetext{
* Correspondence: kawait@kuhp.kyoto-u.ac.jp

'Department of Orthopaedic Surgery, Kyoto City Hospital, 1-2, Higashitakada-cho, Mibu, Nakagyo-ku, Kyoto 604-8845, Japan

${ }^{2}$ Department of Orthopedic Surgery, Kyoto University Graduate School of Medicine, 54 Shogoin-Kawaharacho, Sakyo-ku, Kyoto 606-8507, Japan
}

shortening is utilized in some cases to facilitate reduction, and protect the sciatic nerve.

Several authors reported encouraging results of THA for Crowe IV hips performed using shortening osteotomy [8-10]. However, to the best of our knowledge, few papers have documented the results of THA for Crowe IV hips without shortening osteotomy $[11,12]$. The aim of the present study was to retrospectively assess the long termresults of cemented THAs for Crowe group IV hips performed without subtrochanteric shortening osteotomy.

\section{Methods}

Between August 1992 and November 2004, we performed 38 THAs in 32 patients for Crowe group IV developmental hip dysplasia. Among them, 6 hips (15.8\%) in 5 patients required subtrochanteric shortening osteotomy and were excluded from the study. Shortening osteotomy was performed only when reduction into the true acetabulum seemed otherwise impossible. These six hips were excluded so that the results would be free from any 
influence of shortening osteotomy. Among the remaining 32 hips, 5 were excluded because of short follow-up period of less than 6 years: the 3 patients concerned ( 5 hips) had died of diseases unrelated to their hip problems. This left 27 hips in 24 patients for analysis.

All the procedures were carried out by the senior author (C.T.). The mean age of the patients at the time of the index THA was 57.6 (44 to 80) years. For 23 hips, THA was the first procedure; the remaining 4 hips in 4 patients had previously been treated surgically (femoral osteotomy) before the index THA.

In 16 hips, a CMK (Biomet, Warsaw, IN) cup was used. In the remaining hips, 7 PHS (Kyocera, Kyoto, Japan) cups and 4 small Charnley cups (Thackery, Leeds, UK) were used. The femoral device used was a CMK in 14 hips, and a PHS 6 in 13 hips. A $22 \mathrm{~mm}$ femoral head was always used. Both components were cemented with CMW (DePuy, UK).

The clinical assessments were performed using the Japanese Orthopedic Association (JOA) hip score, which allocates 40 points for pain, 20 points for range of movement, 20 points for walking ability, and 20 points for activities of daily living, with a maximum total score of 100 points. The patients were also examined for the presence of Trendelenburg sign preoperatively and at final follow up.

Radiographic analysis was performed on serial anteroposterior radiographs of the pelvis. On the pelvic side, the presence and evolution of radiolucent lines according to DeLee and Charnley [13] were noted. On the femoral side, investigated parameters included the evolution of radiolucent lines in the 7 zones of the femur [14]. Loosening was defined according to the criteria of Johnston et al. [15] as definite, probable, or possible.

The percentage of structural bone graft for the acetabulum was calculated as the ratio of horizontal length of the graft covering the cup to the horizontal length of the cup (Figure 1). Consolidation and incorporation of the graft was assessed using the criteria of Conn et al. [16] and was defined as identical density of the graft and host bone with a continuous trabecular pattern throughout the graft-host bone junction.

We referenced the location of the femoral head center preoperatively and postoperatively to a line drawn through the teardrops. The vertical height of the femoral head center perpendicular to this reference line was measured. The horizontal distance between the lowest point of the teardrop and femoral head center was measured.

Limb lengthening was defined as the brought down distance of the prominent point of the lesser trochanter that was obtained by comparing the postoperative and preoperative radiographs and corrected according to the magnification. Leg length discrepancy was assessed preoperatively and at final follow up with reference to the lesser trochanter.

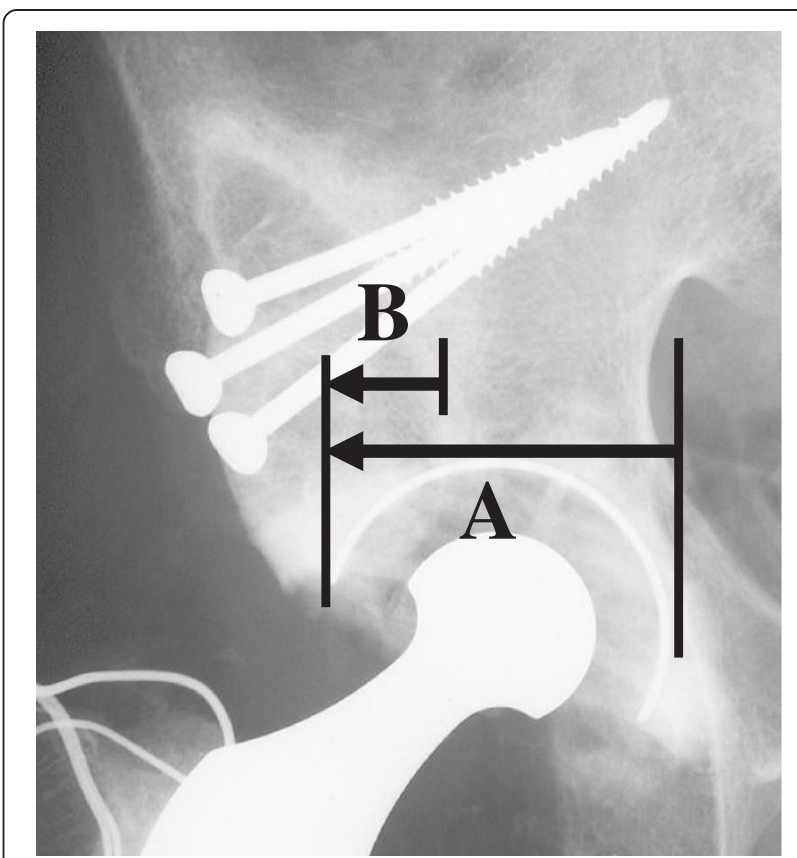

Figure 1 Measurement of percentage of bone graft for the acetabulum, calculated as the ratio of $B$ to $A$.

The study was conducted according to the Declaration of Helsinki [17]. The study protocol was approved by the Ethical Review Board, Kyoto City Hospital, Kyoto, Japan (reference number 188).

\section{Surgical procedure}

THA was carried out in the lateral decubitus position, via the transtrochanteric approach. The joint capsule, fibrous scar tissue, shelf, and osteophytes were removed carefully and completely. The primary landmark to identify was the inferior margin of the acetabulum. After excision of fibrous tissue, the pulvinar, which usually covers the inferior margin of the acetabulum, was recognized as a landmark in all cases. The acetabulum was prepared to create a hemispherical bone cavity with curved gouges and then reamed carefully. A bone autograft obtained from the femoral head and neck was used to augment and reinforce the roof of the undeveloped original acetabulum in all of the 27 procedures. The graft was fixed with at least two screws on the superolateral acetabular defect so that it could cover especially the posterior superior portion of the defect. A socket with an outer diameter of 37 to $40 \mathrm{~mm}$ was cemented into the acetabular cavity.

The trial femoral component was then inserted with the hip dislocated anteriorly and the lower leg aligned vertically, and then the anteversion of the stem was determined in this positioning. After the trial components had been placed, trial reduction was attempted. In high dislocations, soft tissue or neurovascular tension sometimes prevents reduction of the femoral head. When a 
trial reduction was impossible, the additional femoral neck cut was performed until femoral head reduction was possible. Reduction was performed with the hip and the knee both flexed at approximately 30 degrees. It was not achieved by pulling the leg distally but by using a head pusher (Biomet, Warsaw, IN) to push the inserted stem head distally. After reduction, the sciatic nerve tension was assessed by palpation with the knee flexed at approximately 30 degrees. Although reduction was usually tight, muscle release or tenotomy was not performed.

The greater trochanter was reattached with three or four monofilament stainless steel wires in all the hips except three hips; in two hips cable grip and Dall-Miles braided cables were used and in one hip monofilament titanium wires were used instead. The wiring technique consists of three vertical wires and one transverse wire [18]. Patients were free to walk with two supports after two days. Full weight-bearing was usually allowed after six weeks.

\section{Statistical analysis}

Paired t-test was used to assess the change in JOA score. Additionally, considering relatively small sample size, posthoc power analysis was performed to examine the statistical power for the comparison between preoperative and postoperative JOA scores. For statistical analysis, JMP 9 software (SAS Institute, Cary, NC, USA) was used. Differences at $p<0.05$ were considered statistically significant. The endpoint for survival was defined as any revision surgery. Survival was determined using the actuarial life-table constructs described by Kaplan and Meier.

\section{Results}

The mean follow-up period was 10.6 (6.0-17.9) years. The JOA hip score was increased from a mean value of 41.8 (17-65) preoperatively to 80.0 (59-96) at the last followup and this difference was statistically significant $(p<$ 0.001 ). The JOA score improved in all hip. The statistical power for the analysis of difference between preoperative and postoperative JOA score was more than 0.99 .

The Trendelenburg sign was positive in all hips preoperatively and five hips (19\%) at final follow up.

The mean percentage of bone graft for the acetabulum was $32 \%(19-47 \%)$ and mean limb lengthening 3.2 (1.0 to $5.1) \mathrm{cm}$. The leg length discrepancy was $2.7(0-6.8) \mathrm{mm}$ preoperatively and $0.6(0-1.8) \mathrm{cm}$ at final follow up. The mean correction of discrepancy does not exactly match the mean lengthening because in three patients the procedure was performed on both hips for Crowe IV and in 13 patients THA was performed on the contralateral hip for osteoarthritis (Crowe I to III) before final follow up. The rotational center of the hip was reconstructed inferiorly and medially after THA. The average horizontal distance of the postoperative center of the femoral head was 42.3 (30-61) $\mathrm{mm}$ preoperatively and 23.5 (19-34) $\mathrm{mm}$ postoperatively.
The average height of the hip center was 62.5 (51-90) mm preoperatively and $21.7(14-34) \mathrm{mm}$ postoperatively. These results, according to Russotti and Harris [19], indicated that none of the hips had a high hip center, which is defined as more than $35 \mathrm{~mm}$ above the interteardrop line. Figure 2A and $2 \mathrm{~B}$ show radiographs of a case in this series.

Complications were as follows. There were three complications involving the greater trochanter, which included two cases of greater trochanter avulsion resulting from femoral osteolysis in Zone 1 that was caused by debris from the Dall-Miles braided cables used for reattachment of the greater trochanter. There was one case of greater trochanter nonunion with a gap of less than $1 \mathrm{~cm}$, but this did not manifest any symptoms. In this asymptomatic non-union case, monofilament titanium wires had been used for reattachment of the greater trochanter.

Two hips underwent revision surgery: one required isolated stem revision at 13 years after the initial procedure for the abovementioned avulsion of the greater trochanter that occurred subsequent to femoral osteolysis, and the

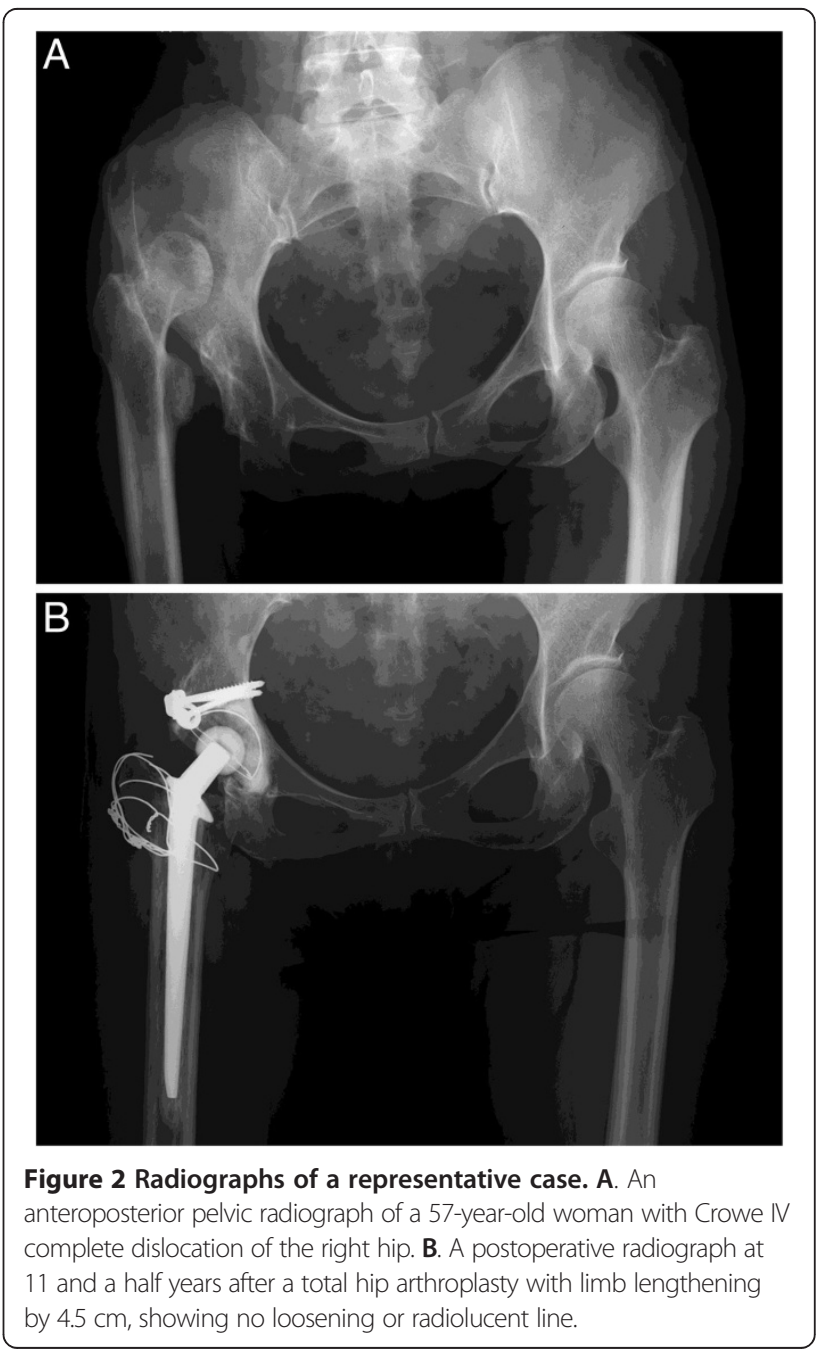


other hip required revision surgery at 18 months after the initial procedure because of early collapse of the acetabular grafted bone. In the latter case, the femoral head used as a graft had been atrophic and possibly too weak, leading to early collapse. This case required a cup revision and reattempted bone grafting, which slightly changed the location of the rotation center and necessitated a stem revision to adjust the limb length. There were no cases of dislocation, infection, or nerve palsy. Among the 27 hips, 6 underwent limb lengthening of more than $4 \mathrm{~cm}$ during the THA. Among those 6 hips, 4 had the abovementioned complications including the two that required revision surgery.

Radiographic analysis demonstrated a partial radiolucent line on the acetabular cement-bone interface of approximately $1 \mathrm{~mm}$ in two hips (one hip in Zone I, the other in Zone III).

Survivorship analysis, with any revision as the endpoint, gave a survival rate of $96.3 \%$ (95\% confidence interval, 76.5 - 99.5\%) at 10 years (Figure 3).

Dall-Miles cables were used in two hips, and these led to complications caused by metal debris from the cables. One hip showed a massive femoral osteolysis in the vicinity of the cable causing avulsion of the greater trochanter and necessitating revision surgery at 13 years after the first procedure. The other hip also had moderate femoral osteolysis leading to avulsion of the greater trochanter; this hip currently remains stable without revision surgery at 15 years of follow-up. In contrast, monofilament stainless wires were used in 24 hips, none of which developed osteolysis or non-union, although one hip suffered early collapse of the bone graft placed on the acetabulum, necessitating cup revision. Monofilament titanium wires were used in one hip, which had trochanteric non-union with a gap of less than $1 \mathrm{~cm}$.

All except one of the acetabulars graft were considered to be united at from 6 to 18 months. One of the graft bones, as mentioned above, gradually collapsed within 18 months postoperatively and required revision surgery.

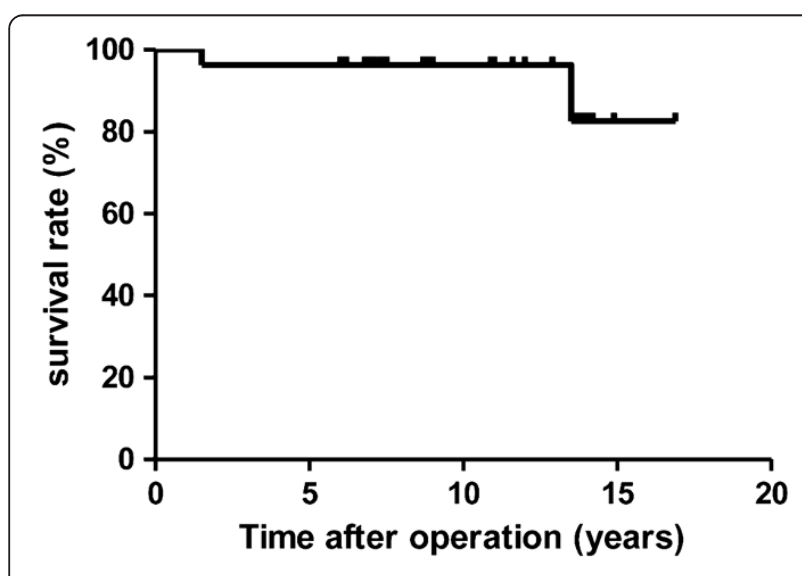

Figure 3 Survivorship with any revision surgery as the endpoint.

\section{Discussion}

Most recent studies of THA for Crowe IV hips have included various types of shortening osteotomy, and these techniques have improved the outcome in such cases. However, there have been few reports on the long-term results of THA with shortening osteotomy [20-22]. Although these results seem encouraging, the long-term outcome at more than 10 years after THAs with shortening osteotomy remains unknown.

Regarding outcomes of THAs without shortening osteotomy for Crowe IV hips, Kerboull et al. reported a survival rate of $78 \%$ at 20 years with revision for any reason as the endpoint [12]. Numair et al. [11] reported higher rates of acetabular revision in completely dislocated hips, as compared with dysplastic hips. Chougle et al. reported that the cup survival rate in Crowe IV hips was $60.9 \%$ at 10 years and $15.6 \%$ at 20 years [23]. Compared with these reports, our results seem satisfactory indicating a survivorship of $96.3 \%$ at 10 years, with any revision as the endpoint.

The most serious risk associated with THA for Crowe group IV hip dislocation is nerve palsy. To avoid this risk, femoral shortening may be required in some cases. In this series, although the leg was lengthened by $3.2 \mathrm{~cm}$ on average, no hips developed nerve palsy. We do not favor muscle release or tenotomy to reduce the hip and always take great care to retain all of the periarticular muscles. The joint capsule, fibrous scar tissue, shelf, and osteophytes were completely removed in the present series. After total capsulectomy psoas muscle can be more extensible. Care was also taken not to pull the leg to reduce the femoral head into the cup, but instead to push the inserted stem head toward the cup center using a head pusher (Biomet, Warsaw, IN) with the hip and the knee both flexed at 30 degrees in order to avoid any excessive traction force on nerves. These approaches may be the reason for the absence of any nerve palsy in the present series.

Another reason to introduce femoral shortening osteotomy in our institute was to avoid problems arising from reattachment of the greater trochanter. The transtrochanteric approach provides wide exposure of the hip, thus facilitating accurate placement of the socket at the level of the true acetabulum in highly dislocated hips. This approach, however, may create problems resulting from reattachment of the greater trochanter when a large amount of limb lengthening is required. Without shortening osteotomy, more extensive resection of the femur to the level of the lesser trochanter is required for reduction in some cases. Extensive resection results in a small trochanteric bed, which may cause pseudoarthrosis of the greater trochanter. We believe that preservation of the trochanteric bed and careful reattachment are important for sound union of the greater trochanter.

Although some authors have reported that limb lengthening should be limited to $4 \mathrm{~cm}$, or even $2 \mathrm{~cm}$, Kerboull 
et al. [12] reported that limb lengthening to $7 \mathrm{~cm}$ was possible. In the present series, bringing the hip down to the level of the true acetabulum and limb lengthening of more than $4 \mathrm{~cm}$ were performed in six hips, none of which developed nerve palsy. These six hips developed four complications necessitating two revisions, whereas 21 the hips with limb lengthening of less than $4 \mathrm{~cm}$ needed no revision, and none developed osteolysis, acetabular loosening, or greater trochanter non-union. These results can be interpreted as indicating that limb lengthening by more than $4 \mathrm{~cm}$ undermines the long-term result even when the rotation center is successfully placed at the level of the true acetabulum without causing nerve palsy. However, among the four complications encountered, three involved problems with the greater trochanter, including two incidences of greater trochanter avulsion accompanied by debris from braided cables leading to massive femoral osteolysis, and one case of stable non-union causing no symptoms and requiring no treatment. These complications suggest that a smaller trochanteric bed resulting from extensive femoral neck resection and the use of braided cables for reattachment of the greater trochanter may be major factors that affect outcomes.

Limb lengthening of $>4 \mathrm{~cm}$ itself does not necessarily lead to problems with the greater trochanter. In our previously reported series of 17 hips that underwent THA via the transtrochanteric approach with subtrochanteric transverse osteotomy, the level of the greater trochanter was lowered by $7.6 \mathrm{~cm}$ on average $(4.3$ to $10.6 \mathrm{~cm}$ ). Although in all 17 hips the greater trochanter was lowered by more than $4.3 \mathrm{~cm}$, no trochanteric non-union or osteolysis occurred [9]. Taken together, these previous results and the present ones suggest that complications involving the greater trochanter result from not only the amount of limb lengthening itself but also the use of braided cables and a smaller trochanteric bed resulting from resection of the femoral neck.

The use of Dall-Miles braided cables to reattach the greater trochanter could have adversely affected the results. Whereas Dall-Miles braided cables were used in two hips, monofilament metal wires were used in the remaining cases. Among the two hips treated with braided cables, one needed stem revision at 13 years after the initial procedure due to massive femoral osteolysis involving the greater trochanter, and the other also showed femoral osteolysis leading to avulsion of the greater trochanter. While several modalities for cerclage fixation are in widespread use, the metal composition imposes potential complications, including third-body generation, and accelerated wear of the bearing surface. Several studies have documented poor outcomes of THAs that were performed with braided cables $[24,25]$. However, we cannot conclude that the use of braided cables had a significant impact on outcome in the present study because of the small number of cases involved. Some of the other major limitations of this study included the retrospective nature of data collection, and the relatively small sample population to measure the survival rate precisely, which resulted in the broad 95\% confidence interval of 76.5 - $99.5 \%$ for 10 -year survival.

\section{Conclusions}

THA for Crowe IV hips without shortening osteotomy provided satisfactory results. However, three out of 27 hips developed complications that involved the greater trochanter. Especially, when braided cables were used to secure the greater trochanter, they led to osteolysis in the long term. Although no nerve palsy occurred in this series, care should always be taken for nerve tension when lengthening the limb.

\section{Competing interests}

The authors declare that they have no competing interest.

\section{Authors' contributions}

TK carried out radiographic analyses and drafted the manuscript. CT performed the operations and revised the manuscript. HK helped to develop the design of the study and contributed to the revision of the manuscript. All authors read and approved the final manuscript.

\section{Authors' information}

Investigation was done in Kyoto City Hospital, but first author currently belongs to Kyoto University.

\section{Acknowledgements}

We gratefully acknowledge Dr. Kanae Kawai Miyake's advice on statistical analysis.

Received: 14 September 2012 Accepted: 4 March 2014

Published: 10 March 2014

\section{References}

1. Malchau $H$, Herberts $P$, Ahnfelt $L$ : Prognosis of total hip replacement in Sweden. Follow-up of 92,675 operations performed 1978-1990. Acta Orthop Scand 1993, 64(5):497-506.

2. Charnley J, Feagin JA: Low-friction arthroplasty in congenital subluxation of the hip. Clin Orthop Relat Res 1973, 91:98-113.

3. Stans AA, Pagnano MW, Shaughnessy WJ, Hanssen AD: Results of total hip arthroplasty for Crowe type III developmental hip dysplasia. Clin Orthop Relat Res 1998, 348:149-157.

4. Pagnano W, Hanssen AD, Lewallen DG, Shaughnessy WJ: The effect of superior placement of the acetabular component on the rate of loosening after total hip arthroplasty. J Bone Joint Surg Am 1996, 78(7):1004-1014

5. Yoder SA, Brand RA, Pedersen DR, O'Gorman TW: Total hip acetabular component position affects component loosening rates. Clin Orthop Relat Res 1988, 228:79-87.

6. Sochart DH, Porter ML: The long-term results of Charnley low-friction arthroplasty in young patients who have congenital dislocation, degenerative osteoarthrosis, or rheumatoid arthritis. J Bone Joint Surg Am 1997 79(11):1599-1617.

7. Crowe JF, Mani VJ, Ranawat CS: Total hip replacement in congenital dislocation and dysplasia of the hip. J Bone Joint Surg Am 1979, 61(1):15-23.

8. Krych AJ, Howard JL, Trousdale RT, Cabanela ME, Berry DJ: Total hip arthroplasty with shortening subtrochanteric osteotomy in Crowe typeIV developmental dysplasia: surgical technique. J Bone Joint Surg Am 2010, 92(Suppl 1 Pt 2):176-187.

9. Kawai T, Tanaka C, Ikenaga M, Kanoe H: Cemented total hip arthroplasty with transverse subtrochanteric shortening osteotomy for Crowe group IV dislocated hip. J Arthroplasty 2011, 26(2):229-235.

10. Makita H, Inaba Y, Hirakawa K, Saito T: Results on total hip arthroplasties with femoral shortening for Crowe's group IV dislocated hips. J Arthroplasty 2007, 22(1):32-38. 
11. Numair J, Joshi AB, Murphy JC, Porter ML, Hardinge K: Total hip arthroplasty for congenital dysplasia or dislocation of the hip. Survivorship analysis and long-term results. J Bone Joint Surg Am 1997, 79(9):1352-1360

12. Kerboull M, Hamadouche M, Kerboull L: Total hip arthroplasty for Crowe type IV developmental hip dysplasia: a long-term follow-up study. $J$ Arthroplasty 2001, 16(8 Suppl 1):170-176.

13. DeLee JG, Charnley J: Radiological demarcation of cemented sockets in total hip replacement. Clin Orthop Relat Res 1976, 121:20-32.

14. Gruen TA, McNeice GM, Amstutz HC: "Modes of failure" of cemented stem-type femoral components: a radiographic analysis of loosening. Clin Orthop Relat Res 1979, 141:17-27.

15. Johnston RC, Fitzgerald RH Jr, Harris WH, Poss R, Muller ME, Sledge CB: Clinical and radiographic evaluation of total hip replacement. A standard system of terminology for reporting results. J Bone Joint Surg Am 1990, 72(2):161-168

16. RA C, LFA P, RN S, D I: Management of acetabular deficiency: long-term results of bone grafting the acetabulum in total hip arthroplasty. Orthop Trans 1985, 9:451-452.

17. Declaration of Helsinki: ethical principles for medical research involvinghuman subjects. In http://www.wma.net/en/30publications/ 10policies/b3/index.html.

18. M K: Arthroplastie totale de hanche sur ankylose. Encyclopedie medicochirurgicale. Techniques chirurgicales-Orthopedie-Traumatolgie. In Encyclopedie medico-chirurgicale Techniques chirurgicales-OrthopedieTraumatolgie. Europe: C Editions Techniques; 1994:44-665.

19. Russotti GM, Harris WH: Proximal placement of the acetabular component in total hip arthroplasty. A long-term follow-up study. J Bone Joint Surg Am 1991, 73(4):587-592.

20. Neumann D, Thaler C, Dorn U: Femoral shortening and cementless arthroplasty in Crowe type 4 congenital dislocation of the hip. Int Orthop 2012, 36(3):499-503.

21. Charity JA, Tsiridis E, Sheeraz A, Howell JR, Hubble MJ, Timperley AJ, Gie GA: Treatment of Crowe IV high hip dysplasia with total hip replacement using the Exeter stem and shortening derotational subtrochanteric osteotomy. J Bone Joint Surg (Br) 2011, 93(1):34-38.

22. Takao M, Ohzono K, Nishii T, Miki H, Nakamura N, Sugano N: Cementless modular total hip arthroplasty with subtrochanteric shortening osteotomy for hips with developmental dysplasia. J Bone Joint Surg Am 2011, 93(6):548-555.

23. Chougle A, Hemmady MV, Hodgkinson JP: Severity of hip dysplasia and loosening of the socket in cemented total hip replacement. A long-term follow-up. J Bone Joint Surg (Br) 2005, 87(1):16-20.

24. Hop JD, Callaghan JJ, Olejniczak JP, Pedersen DR, Brown TD, Johnston RC: The Frank Stinchfield Award. Contribution of cable debris generation to accelerated polyethylene wear. Clin Orthop Relat Res 1997, 344:20-32.

25. Altenburg AJ, Callaghan JJ, Yehyawi TM, Pedersen DR, Liu SS, Leinen JA, Dahl KA, Goetz DD, Brown TD, Johnston RC: Cemented total hip replacement cable debris and acetabular construct durability. J Bone Joint Surg Am 2009, 91(7):1664-1670.

doi:10.1186/1471-2474-15-72

Cite this article as: Kawai et al:: Total hip arthroplasty for Crowe IV hip without subtrochanteric shortening osteotomy -a long term follow up study. BMC Musculoskeletal Disorders 2014 15:72.

\section{Submit your next manuscript to BioMed Central and take full advantage of:}

- Convenient online submission

- Thorough peer review

- No space constraints or color figure charges

- Immediate publication on acceptance

- Inclusion in PubMed, CAS, Scopus and Google Scholar

- Research which is freely available for redistribution

Submit your manuscript at www.biomedcentral.com/submit
C Biomed Central 